\title{
Almost disjoint families and ultrapowers
}

\author{
MichaLIS ANOUSSIS \\ VAGGELIS FELOUZIS \\ KONSTANTINOS TSAPROUNIS
}

\begin{abstract}
We prove estimates for the cardinality of set-theoretic ultrapowers in terms of the cardinality of almost disjoint families. Such results are then applied to obtain estimates for the density of ultrapowers of Banach spaces. We focus on the change of the behavior of the corresponding ultrapower when certain "completeness thresholds" of the relevant ultrafilter are crossed. Finally, we also provide an alternative characterization of measurable cardinals.
\end{abstract}

2020 Mathematics Subject Classification 03C20, 46B08, 03E05, 03 E55 (primary)

Keywords: Ultrafilters, Ultrapowers, Almost disjoint families, Banach spaces, $\varepsilon$-separated sets, Measurable cardinals

\section{Introduction}

The ultrapower technique has a long history, including numerous applications, in modern mathematics and the issue of computing the cardinality of set-theoretic ultrapowers has certainly received much attention (see Keisler [9]). In the present work, motivated by Baumgartner's study in [1], we define an appropriate cardinal function concerning the size of almost disjoint families, which we then use to obtain estimates for the cardinality of such ultrapowers. Our contribution thus suggests the notion of almost disjoint families, with the extensive existing literature regarding them, as a useful toolbox in the context of the ultrapower construction.

We note that for regular ultrafilters, the problem of computing the cardinality of the relevant ultrapowers is completely settled due to a well-known result of Frayne, Morel and Scott (see Theorem 3.1 below). Our results are thus primarily concerned with ultrapowers with respect to non-regular ultrafilters (for example, $\aleph_{1}$-complete), whose existence is well-known to be related with the existence of large cardinals (see, for instance, Ketonen [10] or Magidor [11]). We are, in parallel, interested in the change of the behavior of the corresponding ultrapower when we cross certain "completeness 
thresholds" for the ultrafilter at hand. In particular, we obtain a relevant dichotomy result depending on the completeness number of the ultrafilter.

Given the established estimates for set-theoretic ultrapowers, we then apply our results to obtain estimates for the density of ultrapowers of Banach spaces. In this direction, the notion of an $\varepsilon$-separated set serves as the main tool that enables us to transfer the results from the set-theoretic context.

Finally, we also cast a brief look at Banach ultrapowers via ultrafilters that are (at least) $\aleph_{1}$-complete. Naturally, this involves measurable cardinals; indeed, we provide conditions equivalent to the existence of unboundedly many measurable cardinals and we also prove an alternative characterization of the notion of a measurable cardinal itself. We view this as a further exploration of some results of Ge and Hadwin which appear in $[6, \S 6]$.

\section{Acknowledgement}

The authors would like to thank the anonymous referee for helpful comments and suggestions.

\section{Preliminaries}

We start by giving notational conventions that we will use, as well as some relevant set-theoretic basics. For a comprehensive account, as well as for further details and background material, the reader may consult Jech [7] or Kanamori [8].

Given sets $X$ and $Y$, we write ${ }^{X} Y$ for the set of all functions $f: X \longrightarrow Y$. We write $\omega$ for the set of natural numbers. Given a limit ordinal $\alpha$, we write $\operatorname{cf}(\alpha)$ for its cofinality; if $\kappa$ is a cardinal, then we say that $\kappa$ is regular if $\operatorname{cf}(\kappa)=\kappa$. We denote by GCH the Generalized Continuum Hypothesis.

In what follows, by ultrafilter over $I$ we shall always mean a non-principal ultrafilter over $I$, with the set $I$ always infinite. Given an ultrafilter $\mathcal{U}$ (over some $I$ ), it is sometimes convenient to use the "ultrafilter quantifier" $\forall \mathcal{U}$, which is defined as follows. If $\mathbf{P}(x)$ is any (definable) property with $x$ being a free variable in $\mathbf{P}$, then we say that $\left(\forall \mathcal{U}_{i}\right) \mathbf{P}(i)$ holds if and only if $\{i \in I: \mathbf{P}(i)$ holds $\} \in \mathcal{U}$.

We briefly recall some properties of ultrafilters that we shall use; for more details, the reader is referred to the classical Comfort and Negrepontis [3]. An ultrafilter $\mathcal{U}$ over a 
set $I$ with $|I|=\kappa \geqslant \aleph_{0}$ is called uniform if each $X \in \mathcal{U}$ has size $\kappa$. If $\kappa \geqslant \aleph_{0}$ is a regular cardinal, we say that an ultrafilter $\mathcal{U}$ over the set $I$ is $\kappa$-complete if, for every family $\mathcal{F} \subseteq \mathcal{U}$ with $|\mathcal{F}|<\kappa$, we have that $\bigcap \mathcal{F} \in \mathcal{U}$. Every $\kappa$-complete ultrafilter over (a set of size) $\kappa$ is necessarily uniform.

An ultrafilter $\mathcal{U}$ over an infinite set $I$ is called regular if there exists $\mathcal{F} \subseteq \mathcal{U}$ with $|\mathcal{F}|=|I|$ and so that, for every $i \in I$, the set $\mathcal{F}_{i}=\{X \in \mathcal{F}: i \in X\}$ is finite. Given an infinite set $I$, there is always a regular ultrafilter over $I$.

We say that a cardinal $\kappa>\omega$ is measurable if there is a $\kappa$-complete ultrafilter over $\kappa$. It is well-known (see [7, Lemma 10.2]) that the existence of a measurable cardinal is equivalent to the existence of an $\aleph_{1}$-complete ultrafilter (over some set). We note that the existence of measurable cardinals is not provable from the axioms of ZFC set theory, assuming that $\mathrm{ZFC}$ is consistent.

The completeness number of an ultrafilter $\mathcal{U}$ is the unique cardinal $\kappa$ such that $\mathcal{U}$ is $\kappa$-complete but not $\kappa^{+}$-complete. It follows from standard facts that the completeness number of an ultrafilter is either $\aleph_{0}$ or a measurable cardinal.

Let $X$ be a set and let $\mathcal{U}$ be an ultrafilter over a set $I$. We define an equivalence relation $\sim$ on ${ }^{I} X$ by letting $f \sim g$ if and only if $\left(\forall^{\mathcal{U}} i\right)(f(i)=g(i))$. For each $f \in{ }^{I} X$, we let $[f] \sim=\left\{g \in{ }^{I} X: g \sim f\right\}$. The quotient set:

$$
{ }^{I} X / \sim=\left\{[f] \sim: f \in{ }^{I} X\right\}
$$

will be denoted by $X^{I} / \mathcal{U}$ and will be called the set-theoretic ultrapower of $X$ with respect to $\mathcal{U}$. As a matter of notation, if $|X|=\kappa$ and $|I|=\mu$, then we write $\kappa^{\mu} / \mathcal{U}$ to stand for $\left|X^{I} / \mathcal{U}\right|$, that is, for the cardinality of this ultrapower. For $x \in X$, we let $\operatorname{Id}_{x}$ be the corresponding constant function, namely, $\operatorname{Id}_{x}(i)=x$, for every $i \in I$.

Let $(X,\|\cdot\|)$ be a Banach space and let $\mathcal{U}$ be an ultrafilter over $I$. We say that a function $f \in{ }^{I} X$ is $\mathcal{U}$-bounded if there exists a natural number $n>0$ such that

$$
\{i \in I:\|f(i)\|<n\} \in \mathcal{U}
$$

Now let $\ell_{\infty}(I, X, \mathcal{U})$ be the set of all $\mathcal{U}$-bounded functions $f \in{ }^{I} X$. We define an equivalence relation $\approx$ on $\ell_{\infty}(I, X, \mathcal{U})$ by letting:

$$
f \approx g \Longleftrightarrow \lim _{\mathcal{U}}\|f(i)-g(i)\|=0
$$

Then, the quotient space

$$
\ell_{\infty}(I, X, \mathcal{U}) / \approx=\left\{[f] \approx: f \in \ell_{\infty}(I, X, \mathcal{U})\right\}
$$

will be denoted by $\widetilde{X^{I} / \mathcal{U}}$ and will be called the Banach ultrapower of $X$ with respect to $\mathcal{U}$. The space $\left(\widetilde{X^{I} / \mathcal{U}},\|\cdot\|_{\mathcal{U}}\right)$ is indeed a Banach space, where the norm is $\|[f] \approx\| \mathcal{U}=$ 
$\lim _{\mathcal{U}}\|f(i)\|$. For $a \in X$, considering the elements $\left[\operatorname{Id}_{a}\right]_{\approx}$ of $\widetilde{X^{I} / \mathcal{U}}$, it is clear that $\widetilde{X^{I} / \mathcal{U}}$ contains an isometric copy of the space $X$. Thus, in what follows, we shall freely regard $X$ as a subspace of $\widetilde{X^{I} / \mathcal{U}}$.

\section{Almost disjoint families and the cardinality of set-theoretic ultrapowers}

In this section we provide bounds for the cardinality of set-theoretic ultrapowers in terms of the size of almost disjoint families. Let us note that for regular ultrafilters, the cardinality of the relevant ultrapowers is the largest possible, as given by the following well-known result of Frayne, Morel and Scott:

Theorem 3.1 ([5]) If $\kappa, \lambda$ are infinite cardinals and $\mathcal{U}$ is a regular ultrafilter over $\lambda$, then $\kappa^{\lambda} / \mathcal{U}=\kappa^{\lambda}$.

For non-regular ultrafilters, the general question regarding how the cardinality is affected when passing to ultrapowers is widely open. Nevertheless, we are able to derive some general (lower) bounds, employing the cardinal function $\Psi$ that we define below, which concerns the size of almost disjoint families and which is motivated by Baumgartner's study in [1].

Let $\kappa, \lambda, \mu$ be infinite cardinals such that $\mu \leqslant \kappa$. We shall write $A(\kappa, \lambda, \mu)$ to mean that there exists a family $\mathcal{F}$ of subsets of $\kappa$ such that:

(1) $|\mathcal{F}|=\lambda$

(2) $|a|=\mu$, for every $a \in \mathcal{F}$

(3) $|a \cap b|<\mu$, for every $a, b \in \mathcal{F}$ with $a \neq b$

Informally, $A(\kappa, \lambda, \mu)$ says that $\kappa$ has a family of $\lambda$-many almost disjoint subsets of cardinality $\mu$. Then, for any infinite cardinals $\mu \leqslant \kappa$ we set:

$$
\Psi(\kappa, \mu)=\sup \{\lambda: A(\kappa, \lambda, \mu) \text { holds }\}
$$

Proposition 3.2 Let $\kappa, \mu$ be infinite cardinals with $\mu \leqslant \kappa$. For every uniform ultrafilter $\mathcal{U}$ over $\mu$, we have that $\Psi(\kappa, \mu) \leqslant \kappa^{\mu} / \mathcal{U} \leqslant \kappa^{\mu} \leqslant 2^{\kappa}$.

Proof Let $\lambda$ be any cardinal such that $A(\kappa, \lambda, \mu)$ holds and fix $\mathcal{F}$ a family of subsets of $\kappa$ witnessing this. It is enough to show that $\lambda \leqslant \kappa^{\mu} / \mathcal{U}$. For every $S \in \mathcal{F}$ we fix an injection $f_{S}: \mu \longrightarrow S$. In order to conclude that $|\mathcal{F}| \leqslant \kappa^{\mu} / \mathcal{U}$, we pick 
$S_{1} \neq S_{2}$ any two distinct elements of $\mathcal{F}$ and check that $f_{S_{1}} \nsim f_{S_{2}}$. For this, note that $\left|\left\{i<\mu: f_{S_{1}}(i)=f_{S_{2}}(i)\right\}\right| \leqslant\left|S_{1} \cap S_{2}\right|<\mu$; therefore, using the fact that $\mathcal{U}$ is uniform, we conclude that $f_{S_{1}} \nsim f_{S_{2}}$, as desired.

Using Proposition 3.2 and some facts about the function $\Psi$, all of which follow from results in [1] (see Theorems 2.3, 2.8 and 3.4 there), we immediately obtain the following:

Corollary 3.3 Let $\kappa$ be an infinite cardinal.

(1) Let $\lambda$ be a (possibly finite) cardinal and $\mu$ the least cardinal such that $\lambda^{\mu}>\kappa$. If $\mathcal{U}$ is a uniform ultrafilter over $\mu$ (respectively $\operatorname{cf}(\mu)$ ), then $\kappa^{\mu} / \mathcal{U} \geqslant \lambda^{\mu}$ (respectively $\left.\kappa^{\mathrm{cf}(\mu)} / \mathcal{U} \geqslant \lambda^{\mu}\right)$.

(2) If $2^{<\kappa}=\kappa$ and $\mathcal{U}$ is a uniform ultrafilter over $\kappa$, then $\kappa^{\kappa} / \mathcal{U}=\Psi(\kappa, \kappa)=2^{\kappa}$.

(3) If $\mathcal{U}$ is a uniform ultrafilter over $\kappa$, then $\kappa^{\kappa} / \mathcal{U} \geqslant \kappa^{+}$.

(4) Assume GCH and suppose that $\mu$ is an infinite cardinal with $\mu \leqslant \kappa$ and $\operatorname{cf}(\mu)=\operatorname{cf}(\kappa)$. Then, for every uniform ultrafilter $\mathcal{U}$ over $\mu$, we have that $\kappa^{\mu} / \mathcal{U}=\Psi(\kappa, \mu)=\kappa^{\mu}=2^{\kappa}$.

For historical accuracy, it is important to state that parts of the previous corollary appear in the 1962 work of Frayne, Morel and Scott; namely, part (2) is [5, Lemma 1.24] and [5, Theorem 1.25], while part (3) is [5, Theorem 1.23].

By Theorem 3.1, if $\mathcal{U}$ is a regular ultrafilter over $\kappa$, then $\kappa^{\kappa} / \mathcal{U}=2^{\kappa}$. It follows from part (2) of the previous corollary that we obtain the same conclusion if, instead of assuming regularity for the ultrafilter, one has uniformity (which follows from regularity) and the assumption $2^{<\kappa}=\kappa$.

It is natural to wonder if the equality $\Psi(\kappa, \mu)=\kappa^{\mu} / \mathcal{U}$ holds, more generally, without the GCH or other cardinal-arithmetic assumptions. Let us now show that the answer is independent from the usual axioms of set theory.

Proposition 3.4 The following statement is independent from the ZFC axioms.

(*) If $\mu \leqslant \kappa$ are infinite cardinals with $\operatorname{cf}(\mu)=\operatorname{cf}(\kappa)$ and $\mathcal{U}$ is a uniform ultrafilter over $\mu$, then $\Psi(\kappa, \mu)=\kappa^{\mu} / \mathcal{U}$.

Proof From Corollary 3.3 (4) it follows that GCH implies ( $\star$ ), which means that $(\star)$ is relatively consistent with ZFC. In [1] (see Theorem 5.6 and the discussion following it), a model of ZFC is constructed where $2^{\aleph_{0}}=2^{\aleph_{1}}=\aleph_{3}$ and where $\Psi\left(\aleph_{1}, \aleph_{1}\right)=\aleph_{2}$. In this particular model, we claim that $(\star)$ does not hold. 
For this, assume, towards a contradiction, that $(\star)$ holds in the aforementioned model. Then, for every uniform ultrafilter $\mathcal{U}$ over $\aleph_{1}$ we should have that $\aleph_{1}^{\aleph_{1}} / \mathcal{U}=\aleph_{2}$. On the other hand, let $\mathcal{V}$ be a (any) regular ultrafilter over $\aleph_{1}$. From Theorem 3.1, we must have $\aleph_{1}^{\aleph_{1}} / \mathcal{v}=\aleph_{1}^{\aleph_{1}}=2^{\aleph_{1}}=\aleph_{3}$, a contradiction.

In the next theorem we obtain a dichotomy result for the cardinality of the ultrapower, depending on the completeness number of the ultrafilter.

Theorem 3.5 Let $\kappa, \mu$ be infinite cardinals, let $\mathcal{U}$ be an ultrafilter over $\mu$ and let $\alpha$ be the completeness number of $\mathcal{U}$. Then:

(1) If $\alpha \leqslant \kappa$, then $\kappa^{\mu} / \mathcal{U} \geqslant \Psi(\kappa, \alpha)$.

(2) If $\alpha>\kappa$, then $\kappa^{\mu} / \mathcal{U}=\kappa$.

Proof Of course, note that $\alpha \leqslant \mu$. We first prove (1). Suppose that, for some $\lambda$, we have that $A(\kappa, \lambda, \alpha)$ holds and let $\mathcal{F}$ be a family of subsets of $\kappa$ such that:

(i) $|\mathcal{F}|=\lambda$

(ii) $|S|=\alpha$, for every $S \in \mathcal{F}$

(iii) $\left|S_{1} \cap S_{2}\right|<\alpha$, for every $S_{1}, S_{2} \in \mathcal{F}$ with $S_{1} \neq S_{2}$

At the outset, since $\mathcal{U}$ is not $\alpha^{+}$-complete, we may fix a partition $\left\{Y_{\xi}: \xi<\alpha\right\}$ of the set $\mu$ such that $Y_{\xi} \notin \mathcal{U}$, for all $\xi<\alpha$.

Now let $S \in \mathcal{F}$ be given. Recall that $|S|=\alpha$ (and certainly $S \subsetneq \kappa$ ); so fix an enumeration of $S$ of the form $S=\left\{\gamma_{\xi}: \xi<\alpha\right\}$, where $\gamma_{\xi}$ form a strictly increasing sequence of ordinals below $\kappa$. We define the function $f_{S}$ as follows:

$$
f_{S}=\bigcup_{\xi<\alpha}\left(Y_{\xi} \times\left\{\gamma_{\xi}\right\}\right)
$$

Thus, in order to conclude that $\kappa^{\mu} / \mathcal{U} \geqslant \lambda$, which is enough, we pick $S_{1} \neq S_{2}$ any two distinct elements of $\mathcal{F}$ and we show that $f_{S_{1}} \nsim f_{S_{2}}$.

It is clear that $\left\{i \in \mu: f_{S_{1}}(i)=f_{S_{2}}(i)\right\} \subseteq\left\{i \in \mu: f_{S_{1}}(i) \in S_{1} \cap S_{2}\right\}$. But since $S_{1}, S_{2}$ are distinct members of the family $\mathcal{F}$, we have that $\left|S_{1} \cap S_{2}\right|<\alpha$. Therefore, if $S_{1}$ is enumerated as $S_{1}=\left\{\gamma_{\xi}: \xi<\alpha\right\}$, then, by regularity of $\alpha$, there is $\alpha_{0}<\alpha$ such that $S_{1} \cap S_{2} \subseteq\left\{\gamma_{\xi}: \xi<\alpha_{0}\right\}$, from which it follows that:

$$
\left\{i \in \mu: f_{S_{1}}(i) \in S_{1} \cap S_{2}\right\} \subseteq \bigcup_{\xi<\alpha_{0}} Y_{\xi}
$$

At this point, we recall that $Y_{\xi} \notin \mathcal{U}$, for all $\xi<\alpha$, and that $\mathcal{U}$ is $\alpha$-complete; thus, $\bigcup_{\xi<\alpha_{0}} Y_{\xi} \notin \mathcal{U}$. Therefore, it follows that $\left\{i \in \mu: f_{S_{1}}(i)=f_{S_{2}}(i)\right\} \notin \mathcal{U}$, from which we get that $f_{S_{1}} \nsim f_{S_{2}}$, as desired. 
We now prove (2). For this, it is enough to show that every element $[f]_{\sim}$ of the ultrapower $\kappa^{\mu} / \mathcal{U}$ is equal to some $\left[\operatorname{Id}_{x}\right]_{\sim}$, for some $x \in \kappa$. So let $[f] \sim \in \kappa^{\mu} / \mathcal{U}$, where $f: \mu \longrightarrow \kappa$. For every $x \in \kappa$, we set $A_{x}=\{i \in \mu: f(i)=x\}$ and we note that

$\bigcup A_{x}=\mu \in \mathcal{U}$. Since $\mathcal{U}$ is $\alpha$-complete and $\alpha>\kappa$, there exists $x \in \kappa$ such that $x \in \kappa$

$A_{x} \in \mathcal{U}$. Therefore, we have that $f \sim \operatorname{Id}_{x}$, as desired.

Recall that if $\alpha$ is the completeness number of an ultrafilter, then $2^{<\alpha}=\alpha$; in such a case, by Corollary $3.3(2)$, we have that $\Psi(\alpha, \alpha)=2^{\alpha}$. Thus:

Corollary 3.6 Let $\kappa, \mu$ be infinite cardinals and let $\mathcal{U}$ be an ultrafilter over $\mu$. If the completeness number of $\mathcal{U}$ is $\kappa$, then we have that $\kappa^{\mu} / \mathcal{U} \geqslant 2^{\kappa}$.

Finally, let us mention that, as is well-known, there is extensive literature regarding almost disjoint families and their size. Hence, the interested reader can consult various sources, for instance, Baumgartner [1] or Monk [12], in order to get information about specific values or estimates of the function $\Psi$ in various cases; this way, the results of this section would then produce (more) concrete bounds for the cardinality of the relevant ultrapowers, as in Corollary 3.3.

\section{Density of Banach ultrapowers}

We now apply the results of the previous section in order to obtain estimates for the density of ultrapowers of Banach spaces. We note that all Banach spaces that we will consider are over $\mathbb{R}$ or $\mathbb{C}$.

For a Banach space $(X,\|\cdot\|)$, we let $S_{X}=\{x \in X:\|x\|=1\}$. Moreover, given $\varepsilon \in(0,1)$, a set $A \subseteq S_{X}$ will be called $\varepsilon$-separated if, for every $x, y \in A$ with $x \neq y$, we have that $\|x-y\| \geqslant \varepsilon$.

The density of the space $X$ is the cardinal:

$$
\mathrm{d}(X)=\min \{|D|: D \subseteq X \text { and } \bar{D}=X\}
$$

where $\bar{D}$ denotes the closure of the set $D$. In other words, the density of the space $X$ is the smallest cardinality of a dense subset of $X$. Moreover, if $\varepsilon \in(0,1)$, then the $\varepsilon$-separation number of the space $X$ is the cardinal:

$$
\operatorname{sn}_{\varepsilon}(X)=\sup \left\{|A|: A \subseteq S_{X} \text { and } A \text { is } \varepsilon \text {-separated }\right\}
$$


It is clear that, for every $\varepsilon \in(0,1)$, we have that $\operatorname{sn}_{\varepsilon}(X) \leqslant \mathrm{d}(X)$. The next lemma, which is an easy consequence of Riesz's Lemma, ${ }^{1}$ shows that if $X$ is infinite dimensional, then the supremum in the definition of $\mathrm{sn}_{\varepsilon}$ is attained.

Lemma 4.1 Suppose that $(X,\|\cdot\|)$ is an infinite dimensional Banach space with $\mathrm{d}(X)=\kappa \geqslant \omega$. Then, for every $\varepsilon \in(0,1)$, there exists an $A \subseteq S_{X}$ such that $A$ is $\varepsilon$-separated and $|A|=\kappa$. In particular, we have that $\operatorname{sn}_{\varepsilon}(X)=\mathrm{d}(X)$.

It is convenient to introduce a bit of notation that will be useful below. Let $\mathcal{U}$ be an ultrafilter over the set $I$ and let $X$ be any set. For any $A \subseteq X$, we let:

$$
A^{\mathcal{U}}=\left\{[f]_{\sim} \in X^{I} / \mathcal{U}:\left(\forall^{\mathcal{U}} i\right)(f(i) \in A)\right\}
$$

Similarly, if $X$ is a Banach space and $A \subseteq X$, we let:

$$
\widetilde{A}=\left\{[f] \approx \in \widetilde{X^{I} / \mathcal{U}}:\left(\forall^{\mathcal{U}} i\right)(f(i) \in A)\right\}
$$

When $A=X$, note that $A^{\mathcal{U}}=X^{I} / \mathcal{U}$ and that $\widetilde{A}=\widetilde{X^{I} / \mathcal{U}}$, respectively. If $X$ is a Banach space, it is easy to check that, for any $A \subseteq X$, we have that $|\widetilde{A}| \leqslant\left|A^{\mathcal{U}}\right|$. We will also need the following facts, whose easy proofs we omit:

Lemma 4.2 Let $(X,\|\cdot\|)$ be an infinite dimensional Banach space and $\mathcal{U}$ an ultrafilter over a set $I$.

(1) If $D \subseteq X$ is dense in $X$, then $\widetilde{D}$ is dense in $\widetilde{X^{I} / \mathcal{U}}$.

(2) If $D$ is an $\varepsilon$-separated subset of $X$, for some $\varepsilon \in(0,1)$, then $\widetilde{D}$ is an $\varepsilon$-separated subset of $\widetilde{X^{I} / \mathcal{U}}$.

Lemma 4.3 Let $(X,\|\cdot\|)$ be an infinite dimensional Banach space, $\mathcal{U}$ an ultrafilter over a set $I$ and $A$ an $\varepsilon$-separated subset of $S_{X}$, for some $\varepsilon \in(0,1)$. Then, we have that $|\widetilde{A}|=\left|A^{\mathcal{U}}\right|$.

Proof In this setting, for any functions $f, g \in{ }^{I} X$ such that $\left(\forall^{\mathcal{U}} i\right)(f(i) \in A)$ and $(\forall \mathcal{U} i)(g(i) \in A)$, let us first check that, in fact, $f \approx g$ if and only if $f \sim g$. For the non-trivial direction, suppose that $f \approx g$, let $\delta=\frac{\varepsilon}{2}$ and note that $\{i \in I:\|f(i)-g(i)\|<$ $\delta\} \in \mathcal{U}$. If we let $C=\{i \in I: f(i) \in A$ and $g(i) \in A\}$, then clearly $C \in \mathcal{U}$ and:

$$
\{i \in I: f(i)=g(i)\} \supseteq\{i \in I:\|f(i)-g(i)\|<\delta\} \cap C
$$

\footnotetext{
${ }^{1}$ Recall that Riesz's Lemma says that if $(X,\|\cdot\|)$ is a normed space and $Y$ is a proper closed subspace of $X$, then for every $\varepsilon \in(0,1)$ there is $x \in S_{X}$ such that $\inf \{\|x-y\|: y \in Y\} \geqslant \varepsilon$. For textbook references see, for example, Fabian, Habala, Hájek, Montesinos and Zizler [4, Lemma 1.37] or Riesz and Szőkefalvy-Nagy [13, Lemma 2, page 218].
} 
From this, it immediately follows that $f \sim g$, as claimed.

Now, considering the function $\varphi: A^{\mathcal{U}} \longrightarrow \widetilde{A}$ defined so that, for each $f \in{ }^{I} X$ such that $\left(\forall^{\mathcal{U}} i\right)(f(i) \in A), \varphi\left([f]_{\sim}\right)=[f]_{\approx}$, it is easy to see that $\varphi$ is (well-defined and) bijective, thus proving the desired conclusion.

Using all the above, we now establish a connection between the density of a Banach ultrapower and the cardinality of a relevant set-theoretic ultrapower.

Theorem 4.4 Let $(X,\|\cdot\|)$ be an infinite dimensional Banach space and $\mathcal{U}$ an ultrafilter over a set $I$. Then $\mathrm{d}\left(\widetilde{X^{I} / \mathcal{U}}\right)=\mathrm{d}(X)^{|I|} / \mathcal{U}$.

Proof By Lemma 4.1, for some (fixed throughout) $\varepsilon \in(0,1)$, we can choose an $\varepsilon$-separated subset $D_{1}$ of $X$ with $\left|D_{1}\right|=\mathrm{d}(X)$. Then, from Lemma 4.2, $\widetilde{D_{1}}$ is an $\varepsilon$-separated subset of $\widetilde{X^{I} / \mathcal{U}}$ and so $\mathrm{d}\left(\widetilde{X^{I} / \mathcal{U}}\right) \geqslant\left|\widetilde{D_{1}}\right|$.

Now let $D_{2}$ be a dense subset of $X$ with $\left|D_{2}\right|=\mathrm{d}(X)$. Note that we can assume that $D_{1} \subseteq D_{2}$, since $D_{1} \cup D_{2}$ is also dense with $\left|D_{1} \cup D_{2}\right|=\mathrm{d}(X)$. It thus follows that $\left|\widetilde{D_{1}}\right| \leqslant\left|\widetilde{D_{2}}\right|$. On the other hand, from Lemma 4.3:

$$
\left|\widetilde{D_{1}}\right|=\left|D_{1}^{\mathcal{U}}\right|=\left|D_{2}^{\mathcal{U}}\right| \geqslant\left|\widetilde{D_{2}}\right|
$$

and hence $\left|\widetilde{D_{1}}\right|=\left|\widetilde{D_{2}}\right|$. But recall that, from Lemma 4.2, we have that $\widetilde{D_{2}}$ is a dense subset of $\widetilde{X^{I} / \mathcal{U}}$ and so $\mathrm{d}\left(\widetilde{X^{I} / \mathcal{U}}\right) \leqslant\left|\widetilde{D_{2}}\right|$. Therefore, we have shown that

$$
\left|\widetilde{D_{1}}\right| \leqslant \mathrm{d}\left(\widetilde{X^{I} / \mathcal{U}}\right) \leqslant\left|\widetilde{D_{2}}\right|
$$

from which it now follows that $\left|\widetilde{D_{1}}\right|=\mathrm{d}\left(\widetilde{X^{I} / \mathcal{U}}\right)=\left|\widetilde{D_{2}}\right|$. Finally, since $\left|\widetilde{D_{1}}\right|=\left|D_{1}^{\mathcal{U}}\right|=$ $\mathrm{d}(X)^{|I|} / \mathcal{U}$, we obtain that $\mathrm{d}\left(\widetilde{X^{I} / \mathcal{U}}\right)=\mathrm{d}(X)^{|I|} / \mathcal{U}$, as desired.

Thus, combining Theorem 4.4 with results of Section 3 we immediately get estimates for the quantity $\mathrm{d}\left(\widetilde{X^{I} / \mathcal{U}}\right)$. In particular, invoking Theorem 3.5 , we obtain again a dichotomy result:

Theorem 4.5 Suppose that $(X,\|\cdot\|)$ is an infinite dimensional Banach space with $\mathrm{d}(X)=\kappa$. Let $\mu$ be an infinite cardinal and let $\mathcal{U}$ be an ultrafilter over a set $I$ with $|I|=\mu$. Let $\alpha$ be the completeness number of $\mathcal{U}$. Then:

(1) If $\alpha \leqslant \kappa$, then $\mathrm{d}\left(\widetilde{X^{I} / \mathcal{U}}\right) \geqslant \Psi(\kappa, \alpha)$.

(2) If $\alpha>\kappa$, then $\mathrm{d}\left(\widetilde{X^{I} / \mathcal{U}}\right)=\kappa$. 
It follows that if $\mathcal{U}$ is an $\aleph_{1}$-complete ultrafilter (over a set $I$ ) and $X$ is a separable infinite dimensional Banach space, then $\widetilde{X^{I} / \mathcal{U}}$ is also separable. This is already observed by Ge and Hadwin in [6], following from [6, Proposition 6.1 (2)]. Having said that, we now move on to look at ultrapowers via such "sufficiently complete" ultrafilters, continuing along the line of investigation initiated in $[6, \S 6]$.

\section{Banach ultrapowers and measurable cardinals}

In this final section, we look at Banach ultrapowers via ultrafilters that are (at least) $\aleph_{1}$-complete; naturally, this involves measurable cardinals. In fact, we are going to prove characterizations both for the existence of unboundedly many measurables and for being a measurable cardinal itself.

We shall use the following result, which (independently) appears in Chang and Keisler [2, Proposition 4.2.4] and in Tokarev [14]:

Theorem 5.1 Let $(X,\|\cdot\|)$ be an infinite dimensional Banach space with $\mathrm{d}(X)=\kappa \geqslant \omega$ and let $\mathcal{U}$ be an ultrafilter over a set $I$. The following are equivalent:

(1) $\mathcal{U}$ is $\kappa^{+}$-complete.

(2) The map $e: X \longrightarrow \widetilde{X^{I} / \mathcal{U}}$, sending each $a \in X$ to $\left[\operatorname{Id}_{a}\right]_{\approx}$, is a linear isometry onto $\widetilde{X^{I} / \mathcal{U}}$.

Proof We prove that (1) implies (2). Let $D$ be a dense subset of $X$ with $|D|=\kappa$. It is enough to show that the restricted map $e \uparrow D: D \longrightarrow \widetilde{D}$ is onto since, by Lemma $4.2(1), \widetilde{D}$ is dense in $\widetilde{X^{I} / \mathcal{U}}$. Let $\left\{d_{\xi}: \xi<\kappa\right\}$ be an enumeration of the set $D$ and let $[f] \approx \in \widetilde{D}$, where $f \in{ }^{I} X$. We may assume that $f(i) \in D$ for all $i \in I$. For each $\xi<\kappa$, we let $I_{\xi}=\left\{i \in I: f(i)=d_{\xi}\right\}$. Clearly, $\left\{I_{\xi}: \xi<\kappa\right\}$ is a partition of $I$ and since $\mathcal{U}$ is $\kappa^{+}$-complete, there exists $\xi<\kappa$ such that $I_{\xi} \in \mathcal{U}$. It thus follows that $[f] \approx=e\left(d_{\xi}\right)$, as desired.

We prove that (2) implies (1). Let $\left\{I_{\xi}: \xi<\kappa\right\}$ be a partition of $I$. We shall show that, for some $\xi<\kappa, I_{\xi} \in \mathcal{U}$. For some (fixed throughout) $\varepsilon \in(0,1)$, let $A \subseteq S_{X}$ be an $\varepsilon$-separated set with $|A|=\kappa$. Let $A=\left\{a_{\xi}: \xi<\kappa\right\}$ be an enumeration of $A$. We consider the function $f: I \longrightarrow X$, defined by $f(i)=a_{\xi}$, where $i \in I_{\xi}$. By assumption, we have that $f \approx \operatorname{Id}_{x}$ for some $x \in X$. It follows that $\left\{i \in I:\|f(i)-x\|<\frac{\varepsilon}{2}\right\} \in \mathcal{U}$. Clearly, since $f$ is constant on each $I_{\xi}$, this set is the union of some $I_{\xi}$ 's. But as $A$ is $\varepsilon$-separated, there must be $\xi<\kappa$ such that $\left\{i \in I:\|f(i)-x\|<\frac{\varepsilon}{2}\right\}=I_{\xi}$. Hence, $I_{\xi} \in \mathcal{U}$, concluding the proof. 
Theorem 5.2 The following are equivalent:

(1) There are unboundedly many measurable cardinals.

(2) Let $\kappa$ be a cardinal. There is an ultrafilter $\mathcal{U}$ (over some set $I$ ) such that, for every infinite dimensional Banach space $(X,\|\cdot\|)$ with $\mathrm{d}(X)=\kappa$, the map $e: X \longrightarrow \widetilde{X^{I} / \mathcal{U}}$, sending each $a \in X$ to $\left[\operatorname{Id}_{a}\right]_{\approx}$, is a linear isometry onto $\widetilde{X^{I} / \mathcal{U}}$.

(3) Let $\kappa$ be a cardinal. There is an ultrafilter $\mathcal{U}$ (over some set $I$ ) and an infinite dimensional Banach space $(X,\|\cdot\|)$ with $\mathrm{d}(X)=\kappa$ such that the map $e: X \longrightarrow \widetilde{X^{I} / \mathcal{U}}$, sending each $a \in X$ to $\left[\mathrm{Id}_{a}\right]_{\approx}$, is a linear isometry onto $\widetilde{X^{I} / \mathcal{U}}$.

Proof Clearly (2) implies (3) and it follows from Theorem 5.1 that (1) implies (2). We show that (3) implies (1). Let $\kappa$ be any infinite cardinal and let $\mathcal{U}$ be the ultrafilter given by (3). It follows from Theorem 5.1 that $\mathcal{U}$ must in fact be $\kappa^{+}$-complete. Hence, if $\alpha$ is the completeness number of $\mathcal{U}$, then $\alpha>\kappa$. Therefore, as $\alpha \geqslant \kappa^{+}>\aleph_{0}$ in this case, we conclude that $\alpha$ must be a measurable cardinal above $\kappa$, as desired.

The following final result is a novelty compared to the study in [6], characterizing the measurable cardinal itself, not merely its existence. In the proof below, without loss of generality, we assume for the index set that $I=\kappa$.

Theorem 5.3 Let $\kappa$ be an uncountable cardinal. The following are equivalent:

(1) $\kappa$ is measurable.

(2) The set $\left\{\lambda^{\aleph_{0}}: \lambda<\kappa\right\}$ is an unbounded subset of $\kappa$ and, moreover, there exists an ultrafilter $\mathcal{U}$ over $I=\kappa$ such that, for every infinite dimensional Banach space $(X,\|\cdot\|)$ with $|X|<\kappa$, we have that:

$$
\widetilde{X^{I} / \mathcal{U}}=\left\{\left[\operatorname{Id}_{a}\right]_{\approx}: a \in X\right\}
$$

Proof The fact that (1) implies (2) follows from Theorem 5.1. We show that (2) implies (1). First, recalling that $\left(\lambda^{\aleph_{0}}\right)^{\aleph_{0}}=\lambda^{\aleph_{0}}$, we observe that the assumption on $\kappa$ implies that there are unboundedly many $\lambda<\kappa$ such that $\lambda^{\aleph_{0}}=\lambda$. Thus, it is enough to show that $\mathcal{U}$ is $\lambda^{+}$-complete, for each such $\lambda<\kappa$.

So fix $\lambda<\kappa$ such that $\lambda^{\aleph_{0}}=\lambda$ and let $\left\{A_{\xi}: \xi<\lambda\right\}$ be a partition of $\kappa$ of size $\lambda$. We shall show that there exists some $\xi<\lambda$ such that $A_{\xi} \in \mathcal{U}$. For this, let $X$ be infinite dimensional Banach space with $\mathrm{d}(X)=\lambda$ and note that, by choice of $\lambda$, we have that $|X|=\mathrm{d}(X)^{\aleph_{0}}=\lambda$. Fix some $\varepsilon \in(0,1)$ and let $A \subseteq S_{X}$ be an $\varepsilon$-separated set of size exactly $\lambda$; in fact, let $A=\left\{a_{\xi}: \xi<\lambda\right\}$ be an enumeration of $A$. Moreover, let $\widetilde{X^{I} / \mathcal{U}}=\left\{\left[\operatorname{Id}_{a}\right]_{\approx}: a \in X\right\}$ be the corresponding ultrapower of the space $X$. 
We now consider the function $f: \kappa \longrightarrow A$ defined so that, for each $i<\kappa$ :

$$
f(i)=a_{\xi_{i}}
$$

where $\xi_{i}<\lambda$ is the (unique) ordinal below $\lambda$ such that $i \in A_{\xi_{i}}$. As $[f] \approx \widetilde{X^{I} / \mathcal{U}}$, there exists some $a \in X$ such that $[f]_{\approx}=\left[\mathrm{Id}_{a}\right]_{\approx}$. Then, exactly as in the final part of the proof of Theorem 5.1 (the implication (2) $\Rightarrow(1)$ ), it follows that there exists $\xi<\lambda$ such that $A_{\xi}=\left\{i<\kappa:\|f(i)-a\|<\frac{\varepsilon}{2}\right\} \in \mathcal{U}$, as desired.

Towards concluding, we would like to call attention to the general framework of infinitary languages $\mathcal{L}_{\alpha}$, where $\alpha>\omega$ is a regular cardinal (for more details, see Chang and Keisler [2, Chapter 4, page 230]). In this context, we have a generalized version of Łoś's classical theorem, for ultrapowers via $\alpha$-complete ultrafilters (see [2, Theorem 4.2.11]). Using this machinery, and appealing to the vast expressive power of such languages, it follows that every property of Banach spaces that is expressible in $\mathcal{L}_{\alpha}$, for appropriate $\alpha>\omega$, is preserved in every ultrapower via an $\alpha$-complete ultrafilter. In particular, this provides us with a general setting for directly producing results like the forward direction of Theorem 5.1 or that of the preservation of separability via $\aleph_{1}$-complete ultrafilters, as observed right after Theorem 4.5 above.

Finally, given our dichotomy result Theorem 4.5, let us mention that the general study of ultrapowers in the case in which the ultrafilter has completeness number $\alpha>\omega$ while the Banach space has density greater than or equal to $\alpha$ remains open.

\section{References}

[1] J E Baumgartner, Almost-disjoint sets. The dense set problem and the partition calculus, Ann. Math. Logic 9 (1976) 401-439; https://doi.org/10.1016/0003-4843(76)90018-8

[2] C C Chang, H J Keisler, Model Theory, 3rd edition, Studies in Logic and the Foundations of Mathematics 73, North-Holland (1990); https://doi.org/10.1016/S0049237X(08)70080-6

[3] W W Comfort, S Negrepontis, The Theory of Ultrafilters, Springer-Verlag, Berlin (1974); https://doi.org/10.1007/978-3-642-65780-1

[4] M Fabian, P Habala, P Hájek, V Montesinos, V Zizler, Banach Space Theory, Springer-Verlag, New York (2011); https://doi.org/10.1007/978-1-4419-7515-7

[5] T Frayne, A C Morel, DS Scott, Reduced direct products, Fund. Math. 51 (1962) 195-228; https://doi.org/10.4064/fm-51-3-195-228 
[6] L Ge, D Hadwin, Ultraproducts of $C^{*}$-algebras, In Recent advances in operator theory and related topics, Operator Theory: Advances and Applications 127 (2001) 305-326; https://doi.org/10.1007/978-3-0348-8374-0_17

[7] T Jech, Set Theory, The Third Millennium Edition, Springer-Verlag, Berlin (2003); https://doi.org/10.1007/3-540-44761-X

[8] A Kanamori, The Higher Infinite, Large Cardinals in Set Theory from Their Beginnings, Springer, Berlin (2003); https://doi.org/10.1007/978-3-540-88867-3

[9] HJ Keisler, On cardinalities of ultraproducts, Bull. Amer. Math. Soc. 70 (1964) 644-647; https://doi.org/10.1090/S0002-9904-1964-11219-2

[10] J Ketonen, Nonregular ultrafilters and large cardinals, Trans. Amer. Math. Soc. 224 (1976) 61-73; https://doi.org/10.1090/S0002-9947-1976-0419236-X

[11] M Magidor, On the existence of nonregular ultrafilters and the cardinality of ultrapowers, Trans. Amer. Math. Soc. 249 (1979) 97-111; https://doi.org/10.1090/S0002-99471979-0526312-2

[12] JD Monk, The size of maximal almost disjoint families, Dissertationes Math. 437 (2006) 1-47; https://doi.org/10.4064/dm437-0-1

[13] F Riesz, B Sz.-Nagy, Functional Analysis, Dover Publications, New York (1990)

[14] E Tokarev, Topological and cardinality properties of certain sets of classes of Banach spaces, unpublished preprint (2002); arXiv:0211298

Department of Mathematics, University of the Aegean, Samos 83200, Greece

Department of Mathematics, University of the Aegean, Samos 83200, Greece

Department of Mathematics, University of the Aegean, Samos 83200, Greece

mano@aegean.gr, felouzis@aegean.gr, ktsap@aegean.gr,

kostas.tsap@gmail.com

Received: 13 April 2020 Revised: 30 March 2021 